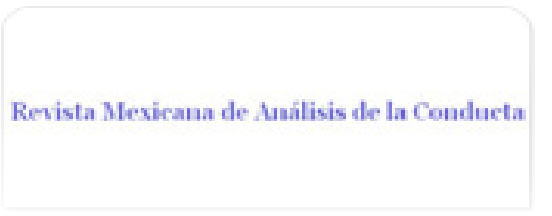

Revista Mexicana de Análisis de la Conducta ISSN: 0185-4534

editora@rmac-mx.org

Sociedad Mexicana de Análisis de la Conducta México

Chaparro Caso López, Alicia Aleli; Morales Chainé, Silvia Análisis de los Tipos de Error en Tareas de Escritura que Son Corregidos a Través de la Tutoría Grupal Entre Compañeros

Revista Mexicana de Análisis de la Conducta, vol. 35, núm. 2, diciembre, 2009, pp. 23-38 Sociedad Mexicana de Análisis de la Conducta

Guadalajara, México

Disponible en: http://www.redalyc.org/articulo.oa?id=59311902003

Cómo citar el artículo

- Número completo

- Más información del artículo

Página de la revista en redalyc.org

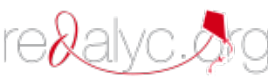

Sistema de Información Científica

Red de Revistas Científicas de América Latina, el Caribe, España y Portugal Proyecto académico sin fines de lucro, desarrollado bajo la iniciativa de acceso abierto 


\title{
Análisis de los Tipos de Error en Tareas de Escritura que Son Corregidos a Través de la Tutoría Grupal Entre Compañeros ${ }^{1}$
}

\author{
Analysis of the Kind of Mistakes on Writing That are
} Correcting Using Class Wide Peer Tutoring

\section{ALICIA ALELI CHAPARRO CASO LÓPEZ Y SILVIA MORALES CHAINÉ UNIVERSIDAD NACIONAL AUTÓNOMA DE MÉXICO}

\begin{abstract}
Resumen
El propósito fue analizar los tipos de error en tareas de escritura que son modificados a través del "Classwide Peer Tutoring" (CWPT). Participaron 2 grupos escolares de segundo grado de primaria quienes fueron evaluados semanalmente a través del dictado de 20 palabras en las que se midió el número de aciertos y el tipo de errores cometidos clasificados de acuerdo con el Inventario de Ejecución Académica (IDEA). Se encontró que todos los errores de regla tuvieron una reducción estadísticamente significativa, y sólo algunos errores específicos se redujeron significativamente. Se encontró además diferencias en el tamaño de la reducción entre los dos grupos participantes. Los resultados se discuten en términos de los alcances que tiene el programa y su relación la literatura previa.
\end{abstract}

Palabras clave: tutoría, niños, errores, escritura, compañeros, tutor, tutorado.

1. Los datos presentados forman parte de la tesis doctoral de la primera autora. La segunda autora colaboró en la obtención de los datos y la redacción del escrito. Dirigir la correspondencia a Alicia Chaparro, Av. Francisco I. Madero No. 24 San Miguel Iztacalco C.P. 08650, México, D.F., o a los correos electrónicos: alicia.chaparro@gmail.com, smchaine@hotmail.com

Recibido: 11 de marzo, 2009. Revisado: 4 de abril, 2009. Aceptado: 21 Octubre, 2009. 


\begin{abstract}
The purpose was to analyze the kind of writing mistakes that are modified by the "Classwide Peer Tutoring" (CWPT) program. Two second grade elementary school groups participated and were evaluated weekly with a dictation of 20 words in which the number of correct answers and the mistakes according to the Academic Execution Inventory (IDEA) were measured. The results were that all rule mistakes were reduced while only some specific mistakes were decreased. In addition a difference in the size of the mistakes reduction between both groups was found. The results are discussed in terms of the program's goals and their relationship to the previous literature.
\end{abstract}

Key words: tutoring, children, mistake, writing, peers, tutor, tutee.

La tutoría entre compañeros de clase es una estrategia académica, que se ha utilizado con éxito para ayudar a que los alumnos con bajo rendimiento mejoren sus calificaciones escolares (Byrd, 1990). Los compañeros tutores en comparación con los maestros utilizan más demostraciones y modelamientos directos, proporcionando asistencia abundante (Kohler \& Greenwood, 1990; Vargas \& Villares, 1999).

El Classwide Peer Tutoring (CWPT, Delquadri, et al, 1986), es un programa de tutoría en el que el tutor no siempre es el estudiante con más alto rendimiento que apoya al estudiante con bajo rendimiento. En este programa, todos los estudiantes pueden realizar los roles de tutor y tutorado, lo que implica que todos tienen la oportunidad de practicar y afianzar sus conocimientos. Además, el CWPT no implica invertir tiempo extra clase para realizar las sesiones de tutoría, pues se realiza diariamente como parte de las actividades escolares, dando oportunidad para que día a día los estudiantes practiquen los conocimientos adquiridos. Por lo tanto, el CWPT es un procedimiento práctico que podría resultar útil dentro de los salones de clases en México, en donde los maestros requieren estrategias que les ayuden a mejorar el aprovechamiento académico de sus estudiantes, permitiéndoles también tener control de grupo y una asignación eficiente del tiempo (Pescador, 1983). Sin embargo, aunque varios estudios han mostrado que el CWPT logra incrementar los resultados académicos de los estudiantes, principalmente en tareas de escritura: "spelling" y dictado (Arreaga-Mayer, 1998; Carta \& Hall, 1986; Delquadri, Greenwood, Whorton,; Greenwood, et al, 1984; Sideridis, et al, 1997), existen también investigaciones que han mostrado que en algunos casos el CWPT no es efectivo.

Kohler y Greenwood (1990) encontraron que sólo 3 de los 4 niños participantes en su estudio mejoraron sus evaluaciones semanales en spelling (pretest $M=26 \%$ vs. postest $M=80 \%$ ). Sin embargo, uno de los niños no mos- 
tró cambios (pretest $M=21 \%$ vs. postest $M=21.4 \%$ ). Además, la cantidad de errores cometidos era una constante que iba incrementando en razón de la cantidad de palabras que el niño iba practicando, concluyendo que el CWPT logró elevar la velocidad con la que el niño podía practicar las palabras, pero no logró corregir las palabras en las que cometió errores desde el inicio.

Mallette, Harper, Mahedy y Dempsey (1991) encontraron que en la aplicación del CWPT en "spelling", hubo palabras que los niños deletreaban de manera incorrecta y que no eran modificadas a través de la tutoría. En las evaluaciones se observó que hubo palabras que los niños ejecutaron de manera incorrecta desde la primera semana de evaluación y continuaron siendo incorrectas en las últimas semanas de aplicación del programa. Mallette et al (1991) concluyeron que es importante llevar a cabo un análisis descriptivo de cómo el CWPT afecta la ejecución de los estudiantes. Una forma de realizar este análisis puede ser revisando los tipos de error que los niños cometen en las evaluaciones académicas.

En todos los estudios en los que se ha probado la efectividad del CWPT, ésta sólo se ha medido a través del número de aciertos que los niños obtienen. Sin embargo, una respuesta incorrecta puede estar formada de uno o varios errores. Por ejemplo, un niño puede escribir "vote" cuando se le dicta la palabra "bote", y esta respuesta es calificada como error. De la misma manera si otro niño escribe "rot" cuando se le dicta "bote" es calificado como error, pero es obvio que los errores cometidos en el primero y segundo ejemplo no son los mismos. Mientras que en el primer caso el niño cometió únicamente un error de sustitución ortográfica sustituyendo la letra "v" por "b" (letras homófonas), en el segundo caso cometió dos errores, por un lado sustituyó la letra "r" por la letra "b" (letras no homófonas) y omitió la última letra de la palabra, teniendo como resultado una palabra que además de ser incorrecta es ilegible y sin sentido. Por lo tanto, los aciertos en sí mismos no proporcionan información suficiente sobre la verdadera efectividad del CWPT.

Según los planteamientos de Eguia (2003) y Macotela, Bermudez y Castañeda (1991) cada tipo de error requiere diferentes tipos de intervención para ser corregido. El niño que no responde correctamente a las demandas académicas puede ser un niño que no haya recibido la instrucción correspondiente, pero también puede ser un niño que no haya aprendido como la mayoría de sus compañeros. En este último caso, el dato de respuesta incorrecta es insuficiente. Es necesario remitirse a los errores que le impidieron responder adecuadamente. Un programa educativo puede tener efectos únicamente sobre ciertos tipos de error mientras que otros errores pueden permanecer constantes debido a que las estrategias educativas del programa pueden no ser funcionales para corregirlos.

El Inventario de Ejecución Académica (IDEA, Macotela, et al, 1991) es un instrumento desarrollado y probado en México que permite evaluar los 
diferentes tipos de error en escritura. Este instrumento clasifica los errores en dos grandes grupos, cada grupo integrado por varios subtipos. El primer grupo de errores corresponde a los errores de regla referentes a un manejo inadecuado de las reglas gramaticales u ortográficas. El segundo grupo lo integran los errores específicos que se refieren a dificultades de tipo discriminativo en donde el niño percibe incorrectamente los estímulos que se le presentan.

Los tipos de error que los niños cometen están relacionados con el nivel de desarrollo de conocimientos y habilidades. Adams, Treiman y Pressley (1998) encontraron que para la adquisición de la lectura los niños requieren desarrollar previamente habilidades como la atención visual y auditiva, la habilidad para distinguir fonemas y símbolos, la comprensión de vocabulario, el desarrollo del lenguaje, etc.

DiLalla, Marcus y Wright-Phillips (2004) y Leppänen, Niemi, Aunola y Nurmi (2004) reportaron que niveles bajos de habilidades preescolares como la conciencia fonológica, la competencia auditiva, la atención visomotora y el nivel educativo materno se relacionan con resultados deficientes en el desarrollo de la alfabetización. Por lo tanto, las habilidades que permiten a los niños aprender a leer y escribir son adquiridas incluso antes del inicio de su vida escolar, por lo que sin una adecuada instrucción los niños pueden mostrar deficiencias académicas a lo largo de su educación (Mathes et al, 2005).

López y Guevara (2008), en un estudio en el que intentaron probar la efectividad de un programa para la prevención de problemas en la adquisición de la lectura y la escritura encontraron que a pesar de que se observaron algunas diferencias entre los grupos control y experimental, el nivel académico obtenido por los alumnos del Grupo Experimental no fue el óptimo después de la intervención, de hecho, fue menor al esperado. Estos autores explicaron este resultado como una función del bajo nivel inicial de las habilidades pre académicas de los alumnos participantes en su estudio.

De la revisión realizada se puede concluir que: a) algunos estudios han mostrado que después de participar en el CWPT los niños continúan cometiendo errores en las evaluaciones académicas, y b) evaluar el éxito de un programa con base únicamente en los aciertos puede resultar insuficiente para analizar los alcances del programa, pues los diferentes tipos de error puede estar asociado con deficiencias en distintas habilidades de los niños y puede requerir procedimientos específicos para ser corregidos. Por lo tanto el propósito de este estudio fue analizar los tipos de error en tareas de escritura que son modificados a través del "Classwide Peer Tutoring" a fin de establecer si el CWPT es efectivo para modificar todos los tipos de error que los niños cometen. 


\section{MÉTODO}

\section{Participantes}

Participaron dos grupos escolares de segundo grado de primaria de una escuela pública de la Ciudad de México. Se eligieron niños de segundo grado debido a que se asume que en este nivel escolar los niños ya aprendieron las reglas básicas de la escritura de las palabras, y por lo tanto los errores que se identificarían en las pruebas, no estarían asociados al desconocimiento de las reglas de escritura (Flórez, Arias y Guzmán, 2006). El grupo $2^{\circ} \mathrm{A}$ estuvo formado por 29 niños, 14 niñas y 15 niños con un promedio de edad de 7 años 6 meses y un promedio escolar de 8.39 (rango 5 a 9 ). El grupo $2^{\circ} \mathrm{B}$ estuvo formado por 29 niños, 12 niñas y 17 niños con un promedio de edad de 7 años 4 meses y un promedio escolar de 8.89 (rango 6 a 9.8). Participaron también las 2 maestras responsables de cada grupo escolar. La maestra de $2^{\circ} \mathrm{A}$ tenía 47 años y 18 años de experiencia docente. La maestra de $2^{\circ} \mathrm{B}$ tenía 53 años y 23 años de experiencia docente. Como criterio de selección se estableció eliminar a los estudiantes que tuvieran algún tipo de disfunción física (impedimento físico para escribir) o perceptual (cualquier problema visual o auditivo). Ninguno de los niños de los 2 grupos escolares cubrió este criterio por lo que todos fueron incluidos en la muestra.

\section{Instrumentos y Materiales}

Se aplicaron pruebas semanales en las que cada maestra dictaba a los niños de su grupo 20 palabras de 2 a 4 sílabas (ver Anexo 1). Las palabras que se dictaron en cada prueba eran elegidas de común acuerdo por las dos maestras con base en el plan de trabajo escolar. La misma lista de palabras fue utilizada en los grupos $2^{\circ} \mathrm{A}$ y $2^{\circ} \mathrm{B}$. Una vez que el dictado de las palabras eran concluido, las pruebas eran recogidas por las maestras y entregadas a dos estudiantes de psicología previamente entrenados, quienes las calificaban identificando aciertos y tipos de error cometidos. En cada prueba se identificaron los aciertos y errores de acuerdo con el protocolo de evaluación del Inventario de Ejecución Académica (IDEA, Macotela et al, 1991). Un acierto se consideraba cuando había ausencia de cualquier tipo de error. Los errores de regla eran modificaciones de la escritura que alteraban la ortografía de la palabra, pero que no alteraban su significado ni sonido. Los errores específicos eran modificaciones que alteraban además de la ortografía, la semántica y fonética de la palabra. Una misma palabra podía presentar más de un tipo de error. En el anexo 2 se puede revisar la definición de cada subtipo de error. 


\section{Procedimiento}

Se utilizó un diseño experimental A-B con réplica, usado en varios estudios del CWPT (Arreaga-Mayer, 1998), y en el que la fortaleza de los resultados está dada por la réplica de los mismos (Cooper, Heron y Heward, 1987). La línea base consistió en la utilización del procedimiento habitual para la escritura correcta de las palabras, en donde los niños hacían planas de repaso o las maestras les dictaban de forma repetida las palabras. La fase de intervención consistió en la utilización del CWPT.

Para llevar a cabo la aplicación del CWPT (Delquadri et al, 1986) fue necesario entrenar simultáneamente a ambas maestras. Durante el entrenamiento se utilizó material escrito, exposición oral de los procedimientos, modelamiento, ensayo y retroalimentación. El entrenamiento se realizó durante la $3^{\text {a }}$ semana de línea base ocupando los 30 minutos del recreo. Durante la $4^{\mathrm{a}}$ semana de línea base cada maestra llevó a cabo el entrenamiento de sus alumnos. Las sesiones de entrenamiento tuvieron una duración de 30 minutos. Aunque el entrenamiento de los niños se realizó estando aún en la fase de línea base, su entrenamiento no afecto los resultados de las evaluaciones pues sólo fueron entrenados en el procedimiento del proceso de tutoría pero no lo utilizaron para practicar el aprendizaje de la semana, por lo que el entrenamiento no afecto los resultados de las evaluaciones de los alumnos

En el entrenamiento a los alumnos las maestras mostraron a través de modelamiento y ensayo conductual que el trabajo de tutoría se realizaba en parejas que se asignaban a equipos. En cada equipo había de 7 a 8 parejas aproximadamente. Las parejas eran formadas heterogéneamente según su rendimiento académico. Las parejas eran formadas por las maestras y se asignaban aleatoriamente a uno de los dos equipos. Las parejas y equipos eran reasignados cada semana.

Los nombres de las parejas que integraban cada equipo eran colocados en carteles en una parte visible del salón, de modo que los niños podían revisar por ellos mismos el compañero con el que formarían pareja para trabajar durante la semana. En cada pareja los papeles de tutor y tutorado se intercambiarían en la misma sesión. A través del apoyo de un modelo escrito, el tutor dictaba las palabras a su compañero tutorado. Por cada palabra escrita correctamente el tutor otorgaba 2 puntos a su tutorado y 1 punto si la escriba incorrectamente pero la corregía y repetía 3 veces. Los puntos que cada tutorado acumulaba eran sumados a los del resto del equipo y al final de la sesión de tutoría (generalmente de 30 minutos) se nombraba a un equipo ganador. El equipo ganador sólo ganaba el reconocimiento social del otro equipo quien les aplaudían después de ser nombrado como equipo ganador. Dentro de la semana de entrenamiento a los niños y en las semanas posteriores, en cada salón estuvo presente un estudiante de psicología, 
quien además de observar el apego de las maestras a los procedimientos del CWPT, las auxiliaba cuando había dudas u olvido de lo que debían realizar.

\section{RESULTADOS}

Para realizar el análisis de los resultados, en cada grupo se promediaron los aciertos y tipos de error que los niños obtuvieron en las pruebas semanales. A fin de evaluar si los datos de la línea base y de la aplicación del CWPT mostraban diferencias estadísticamente significativas se utilizó una prueba no paramétrica para muestras relacionadas (Wilcoxon). Se utilizó estadística no paramétrica dada la variabilidad de los resultados de cada grupo. Los resultados mostraron que ambos grupos tuvieron un incremento en el número de aciertos una vez que el CWPT fue implementado. En el grupo $2^{\circ} \mathrm{A}$ el porcentaje promedio de aciertos en la línea base fue de $26 \%$ y se incrementó a $57.8 \%$ durante el CWPT $[Z=-4.70, p<.01]$. En el grupo $2^{\circ} \mathrm{B}$, el promedio de aciertos durante la línea base fue de $49.30 \%$ incrementando a $80.34 \%$ durante la fase del CWPT [ $Z=4.70, p<.01]$.

En la Tabla 1 se muestran las medias y desviaciones estándar (entre paréntesis) de los subtipos de error de regla. Puede observarse que el CWPT redujo significativamente todos los subtipos de error de regla en ambos grupos escolares. Sin embargo vale la pena analizar las diferencias en la frecuencia de ocurrencia que muestran los diferentes subtipos en cada grupo. . En $2^{\circ} \mathrm{A}$ el subtipo con más frecuencia fue la omisión ortográfica, mostrando reducción entre la fase de línea base $(M=4.47)$ y CWPT $(M=2.06)$. En el grupo $2^{\circ} \mathrm{B}$ el subtipo con mayor frecuencia fue la sustitución ortográfica, el cual mostró también una reducción entre línea base $(M=3.96)$ y el CWPT $(M=1.30)$. La sustitución de mayúsculas por minúsculas fue el subtipo de error de regla con menor frecuencia en ambos grupos pero fue el que presentó la mayor reducción. En el grupo $2^{\circ} \mathrm{A}$ tuvo un promedio por semana de 0.95 en línea base y 0.18 durante el CWPT. En el grupo $2^{\circ} \mathrm{B}$ el promedio por semana durante línea base fue de 1.58 y 0.19 durante el CWPT. 
Tabla 1

Comparación entre LB y CWPT de los promedios y las desviaciones estándar de las frecuencias de errores de regla

\begin{tabular}{lcccccc}
\hline Tipo de error & \multicolumn{5}{c}{$2^{\circ} \mathrm{A}$} & \multicolumn{4}{c}{$2^{\circ} \mathrm{B}$} \\
\cline { 2 - 7 } & $\mathrm{LB}$ & $\mathrm{CWPT}$ & $\mathrm{Z}$ & LB & CWPT & $\mathrm{Z}$ \\
\hline Sustitución ort. & $3.43(1.10)$ & $1.33(1.33)$ & $-4.62^{* *}$ & $3.96(1.58)$ & $1.30(1.05)$ & $-4.60^{* *}$ \\
Omisión ort. & $4.47(2.63)$ & $2.06(2.41)$ & $-4.64^{* *}$ & $1.49(1.34)$ & $0.64(0.67)$ & $-319^{* *}$ \\
O. de acentos & $2.39(1.06)$ & $1.55(1.12)$ & $-2.79^{* *}$ & $3.05(1.18)$ & $1.16(0.83)$ & $-4.45^{* *}$ \\
Sust. May/min & $0.95(0.46)$ & $0.18(0.30)$ & $-4.48^{* *}$ & $1.58(0.75)$ & $0.19(0.26)$ & $-4.63^{* *}$ \\
\hline
\end{tabular}

${ }^{* *} p<.01$

Las diferencias encontradas tanto en la frecuencia de ocurrencia de los errores en ambos grupos, como en la cantidad de errores que se redujeron durante el CWPT, hizo necesario analizar si la cambio fue igual en ambos grupos. Para obtener el porcentaje de cambio, es decir el porcentaje de incremento en los aciertos y el porcentaje de reducción en los errores, se aplicó la siguiente fórmula:

\section{Porcentaje de cambio $=(M$ LB- $M$ CWPT $) / M L B \times 100$}

En donde el promedio de la línea base se restó al promedio del CWPT y este resultado se dividió entre el promedio de la línea base multiplicándolo por 100 , obteniendo así el porcentaje del incremento o reducción. A través de esta fórmula se encontró que en el grupo $2^{\circ} \mathrm{A}$ el porcentaje del incremento en los aciertos fue de $157.78 \%$ y en el grupo $2^{\circ} \mathrm{B}$ el porcentaje del incremento fue de $87.50 \%$. Esta diferencia en el porcentaje del incremento de aciertos entre ambos grupos resultó estadísticamente significativa $[Z=-2.5, p<.05]$. Sin embargo aunque el porcentaje del incremento de aciertos es mayor en el grupo $2^{\circ} \mathrm{A}$ que en $2^{\circ} \mathrm{B}$, el porcentaje en la reducción de errores resulto mayor en $2^{\circ} \mathrm{B}$.

En la Figura 1 se muestran los porcentajes de reducción de cada uno de los subtipos de error de regla en ambos grupos escolares. Para analizar las diferencias entre ambos grupos se utilizó la prueba de Mann-Whitney. Se puede observar que en todos los subtipos de error el porcentaje de reducción es mayor en el grupo de $2^{\circ} \mathrm{B}$ que en $2^{\circ} \mathrm{A}$. Con excepción de la sustitución ortográfica, todos los subtipos de error de regla mostraron diferencias estadísticamente significativas en los porcentajes de reducción entre $2^{\circ} \mathrm{A}$ y $2^{\circ} \mathrm{B}$. En el grupo $2^{\circ} \mathrm{A}$, la omisión ortográfica mostró una reducción de $53.91 \%$ mientras que en $2^{\circ} \mathrm{B}$ la reducción fue de $57.04 \%$ [ $\left.Z=-4.08, p<.001\right]$. La omisión de acentos tuvo una reducción de $35.14 \%$ en $2^{\circ} \mathrm{A}$, y en $2^{\circ} \mathrm{B}$ la reducción fue de $61.96 \%,[Z=-2.69, p<.001]$. Finalmente la sustitución de mayúsculas 
por minúsculas tuvo una reducción de $81.05 \%$ en $2^{\circ} \mathrm{A}$ y de $87.97 \%$ en $2^{\circ} \mathrm{B}$, $[Z=-3.25, p<.001]$.

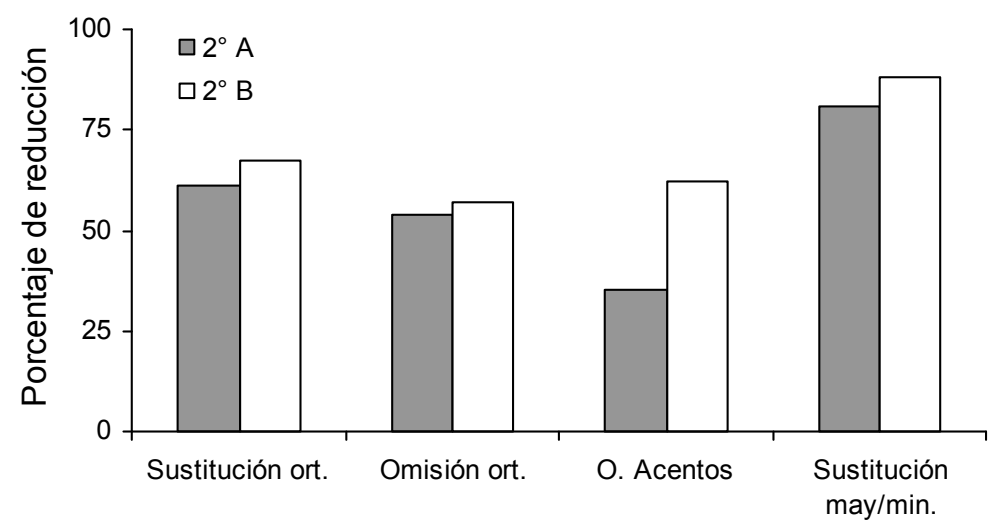

Figura 1. Se muestran los porcentajes de reducción de los errores de regla en los grupos de $2^{\circ} \mathrm{A}$ y $2^{\circ} \mathrm{B}$. Las barras representan los promedios grupales de 29 niños en cada grupo.

Los subtipos de error específico se muestran en la Tabla 2. Se observa que en el grupo $2^{\circ} \mathrm{A}$ todos los subtipos de error con excepción de la omisión tuvieron una reducción estadísticamente significativa. En grupo $2^{\circ} \mathrm{B}$ sólo la sustitución y la omisión tuvieron una reducción estadísticamente significativa. Sin embargo en este grupo transposición y la inversión aunque no mostraron una reducción significativa, tuvieron una baja frecuencia en línea base y en el CWPT su frecuencia llegó a cero ocurrencias. También se observa que la frecuencia de los subtipos de error en cada grupo es diferente. Se observa que únicamente la sustitución y la omisión tuvieron una reducción estadísticamente significativa en ambos grupos. La sustitución tuvo un promedio de ocurrencia en $2^{\circ} \mathrm{A}$ de 1.81 durante línea base y se redujo a 0.70 durante el CWPT, mientras que en el grupo $2^{\circ} \mathrm{B}$ tuvo un promedio de 0.92 reduciéndose a 0.17 en el CWPT. La omisión en el grupo $2^{\circ} \mathrm{A}$ tuvo un promedio de 0.98 en línea base reduciéndose a 0.56 durante el CWPT y en $2^{\circ} \mathrm{B}$ tuvo un promedio de 0.31 en línea base y 0.04 en CWPT. La inversión fue el subtipo de error que ocurrió con menor frecuencia, en el grupo $2^{\circ} \mathrm{A}$ tuvo una frecuencia de 0.24 durante línea base y 0.001 durante CWPT resultando esta reducción estadísticamente significativa. En $2^{\circ} \mathrm{B}$ tuvo una frecuencia de 0.02 durante línea base reduciéndose a cero durante el CWPT. 
Tabla 2

Comparación entre LB y CWPT de los promedios y las desviaciones estándar de las frecuencias de errores específicos

\begin{tabular}{|c|c|c|c|c|c|c|}
\hline \multirow[t]{2}{*}{ Tipo de error } & \multicolumn{3}{|c|}{$2^{\circ} \mathrm{A}$} & \multicolumn{3}{|c|}{$2^{\circ} B$} \\
\hline & $L B$ & CWPT & Z & $L B$ & CWPT & Z \\
\hline Sustitución & $1.81(2.38)$ & $0.70(1.10)$ & $-3.59^{* *}$ & $0.92(0.78)$ & $0.17(0.31)$ & $-3.87^{* *}$ \\
\hline Omisión & $0.98(1.57)$ & $0.56(1.18)$ & -1.95 & $0.31(0.48)$ & $0.04(0.11)$ & $-2.83^{* *}$ \\
\hline Adición & $0.45(0.51)$ & $0.15(0.30)$ & $-2.19^{* *}$ & $0.17(0.32)$ & $0.09(0.21)$ & -1.34 \\
\hline Transposición & $0.15(0.33)$ & $0.01(0.06)$ & $-2.38^{*}$ & $0.01(0.04)$ & 0.00 & -1.00 \\
\hline Inversión & $0.24(0.70)$ & $0.01(0.04)$ & $-2.03^{*}$ & $0.02(0.13)$ & 0.00 & -1.00 \\
\hline
\end{tabular}

Los porcentajes de reducción de los subtipos de error específico se muestran en la Figura 2. Se puede observar que todos los subtipos de error mostraron una reducción mayor en el grupo $2^{\circ} \mathrm{B}$ que en $2^{\circ} \mathrm{A}$. Aunque la $\mathrm{Fi}$ gura 2 muestra diferencias notables a nivel visual en la sustitución, omisión e inversión, únicamente la adición y la transposición mostraron diferencias estadísticamente significativas. La adición tuvo una reducción de $26.72 \%$ en $2^{\circ} \mathrm{A}$ y $62.06 \%$ en $2^{\circ} \mathrm{B},[Z=-1.96, p<.05]$. La transposición se redujo un $93.33 \%$ en $2^{\circ} \mathrm{A}$ y un $100 \%$ en $2^{\circ} \mathrm{B},[Z=-2.31, p<.05]$.

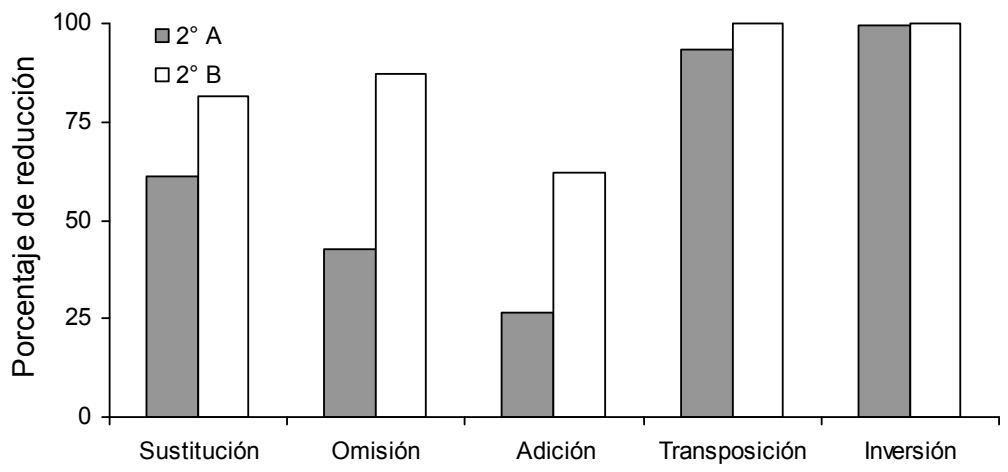

Figura 2. Se muestran los porcentajes de reducción de los errores específicos en los grupos de $2^{\circ} \mathrm{A}$ y $2^{\circ} \mathrm{B}$. Las barras representan los promedios grupales de 29 niños en cada grupo. 
Los resultados mostraron que los errores de regla ocurrieron con mayor frecuencia que los errores específicos en ambos grupos. Los errores de regla tuvieron una reducción estadísticamente significativa en ambos grupos. Mientras que sólo en algunos de los errores específicos la reducción fue estadísticamente significativa.También se encontró que existieron diferencias en los porcentajes de reducción entre ambos grupos.

\section{DISCUSIÓN}

Los resultados mostraron que el CWPT incrementó el porcentaje de aciertos en las pruebas semanales de escritura de los grupos de $2^{\circ} \mathrm{A}$ y $2^{\circ} \mathrm{B}$, replicado los resultados de estudios anteriores donde fue utilizado (Arreaga-Mayer, 1998, Sideridis et al, 1997, Delquadri, et al, 1986, Greenwood, et al, 1984). Sin embargo, hubo una diferencia significativa en el porcentaje del incremento de los aciertos entre ambos grupos, lo que indicó que el CWPT resultó más efectivo en el grupo $2^{\circ} \mathrm{A}$ que en el grupo $2^{\circ} \mathrm{B}$. No obstante, al analizar en detalle estos resultados se observó que aunque el porcentaje del incremento fue mayor en $2^{\circ} \mathrm{A}$ que en $2^{\circ} \mathrm{B}$, la reducción en el número de errores que los niños cometieron fue al revés, es decir hubo una mayor reducción de errores tanto de regla como específicos en $2^{\circ} \mathrm{B}$ que en $2^{\circ} \mathrm{A}$. Este resultado fundamenta la afirmación de que analizar los errores que son corregidos a través del CWPT es una forma alternativa de probar la efectividad de dicho programa y de cualquier otro programa educativo, pues como se observó los aciertos son sólo ausencia de error, pero un error no es proporcional a un acierto, es decir una palabra calificada como incorrecta puede estar compuesta de varios errores.

En el análisis de los errores cometidos, se pudo observar que en ambos grupos los niños cometieron más errores de regla que errores específicos. También se observó que el CWPT disminuyó significativamente todos los errores de regla en ambos grupos, pero algunos errores específicos no mostraron reducciones estadísticamente significativas. Por ejemplo sólo la sustitución y la omisión mostraron una reducción significativa en ambos grupos escolares. Por el contrario, la adición y la transposición se redujeron significativamente sólo en el grupo $2^{\circ} \mathrm{A}$ y la inversión no tuvo una reducción estadísticamente significativa en ninguno de los grupos.

Los hallazgos en la diferencia de los efectos en los errores de regla y específicos se vinculan con los planteamientos de Eguia (2003) y Macotela et al (1991) quienes señalaron que cada tipo de error requiere diferentes tipos de intervención para ser corregido. Los resultados de este estudio mostraron que aunque los errores específicos ocurrieron en menor frecuencia que los errores de regla, no tuvieron el mismo nivel significativo de reducción, indi- 
cando que el CWPT no reduce en la misma proporción los diferentes tipos de error y que los errores específicos pueden requerir algún tipo particular de intervención.

Macotela et al (1991) señalaron que los errores de regla se relacionan con un manejo inadecuado de las reglas gramaticales u ortográficas, es decir son errores más bien asociados a la práctica que los niños tienen para el aprendizaje de estas reglas. EI CWPT es un programa que permite a los niños practicar de manera reiterativa las reglas gramaticales y ortográficas, de ahí el que se hayan encontrado diferencias estadísticamente significativas en la reducción de estos errores en ambos grupos. Por otro lado, los errores específicos que se refieren a dificultades de tipo discriminativo en donde el niño altera la percepción de los estímulos que se le presentan se relacionan directamente con las habilidades previas que los niños necesitan desarrollar para el adecuado aprendizaje de la lecto-escritura. En este caso los errores específicos que no muestran el mismo nivel de cambio no fueron reducidos significativamente. Adams et al (1998) señaló que la atención visual y auditiva, la habilidad para distinguir fonemas y símbolos y la comprensión de vocabulario son necesarios para el aprendizaje de la lecto-escritura. Todas estas habilidades se asocian directamente con la percepción de los estímulos y por ende con la ocurrencia de errores específicos.

Se esperaría que dada la similitud que ambos grupos tenían entre sí al pertenecer a la misma escuela, tener el mismo número de alumnos y promedios escolares similares, la reducción de los errores fuera igual en ambos grupos. Sin embargo, la diferencia en los aciertos obtenidos desde la línea base, indicó que eran diferentes entre sí, contando probablemente con un nivel diferente de conocimiento y habilidades, lo que hizo necesario analizar si esa diferencia marcaba también una diferencia estadísticamente significativa en los ciertos era también significativa en los errores. Los análisis permitieron afirmar que los niños del grupo $2^{\circ} \mathrm{B}$ contaban con conocimientos y/o habilidades que le permitieron alcanzar mejores resultados con la utilización del CWPT, Estos resultados parecen confirmar los hallazgos de otros estudios que mostraron que la adquisición de la lecto-escritura depende de ciertas habilidades que son precurrentes y que los niños desarrollan antes del inicio de su vida escolar o durante la etapa preescolar (Adams, et al, 1998; DiLalla et al, 2004; Leppänen et al, 2004). No obstante aunque era evidente que ambos grupos escolares eran diferentes entre sí, es importante en un estudio posterior evaluar los conocimientos y habilidades que los niños tienen antes de participar en el CWPT para poder establecer esta conclusión de manera contundente.

Como primer conclusión se puede afirmar que el CWPT no incrementó en la misa proporción los resultados académicos de los niños. Haber analizado los efectos del CWPT sobre los diferentes tipos de error permitió conocer 
que este programa no afecta de la misma manera los errores de regla que los errores específicos. Por lo tanto una forma de probar la efectividad de un programa educativo no sólo es medirlo a través de los cambios en el número de aciertos sino también en los tipos de error que los niños cometen. Estos resultados son acordes a los planteamientos de Eguia (2003) y Macotela et al (1991) quienes señalaron que cada tipo de error requiere diferentes tipos de intervención para ser corregido. El dato de respuesta incorrecta es insuficiente. Es necesario revisar los errores que le impidieron responder adecuadamente. Un programa educativo puede tener efectos únicamente sobre ciertos tipos de error mientras que otros errores pueden permanecer constantes tal como ocurrió con los errores específicos en el presente estudio.

Para finalizar, se puede decir que los análisis realizados permitieron probar de una forma alternativa la efectividad del CWPT, y mostraron que analizar su efectividad únicamente a través de los aciertos puede resultar insuficiente. Aunque se replicaron los resultados de otras investigaciones en las que se ha encontrado que el CWPT incrementa el porcentaje de aciertos, también se encontró que los aciertos no son la única forma de analizar los efectos de este programa. Se pudo constatar que el análisis de los errores arrojó información más descriptiva, notándose que no hubo los mismos efectos en los diferentes tipos de error. Además se pudo también probar que la reducción en los errores que los niños cometen parece relacionarse no sólo con la aplicación del programa, sino con el conocimiento previo y/o habilidades con las que estos cuenten al momento de iniciar su participación y como se mencionó anteriormente es necesario realizar un estudio posterior donde se puedan evaluar de que manera los conocimientos y/o habilidades previas de los niños afectan los resultados de la intervención.

\section{REFERENCIAS}

Adams, M. J., Treiman, R., \& Pressley, M. (1998). Reading, writing and literacy. En: W. Damon, I. E. Sigel, \& K. A. Renninger, (Eds.). Handbook of child psychology. Child psychology in practice. (pp. 275-355). New York: John Wiley \& Sons Inc.

Arreaga-Mayer, C. (1998). Increasing active student responding and improving academic performance through classwide peer tutoring. Intervention in School \& Clinic, 34, 89-95.

Byrd, D. E. (1990). Peer tutoring with the learning disabled: A critical review. Journal of Educational Research, 84, 115-118.

Cooper, J. O., Heron, T. E., \& Heward, W. L. (1987). Applied Behavior Analysis. Columbus, $\mathrm{OH}$ : Merrill.

Delquadri, J., Greenwood, C. R., Whorton, D., Carta, J. J., \& Hall, R. V. (1986). Classwide peer tutoring. Exceptional Children, 52, 535-542. 
DiLalla, L. F., Marcus, J. L., \& Wright-Phillips, M. V. (2004). Longitudinal effects of preschool behavioural styles on early adolescent school performance. Journal of School Psychology, 42, 385-401.

Eguia, M. S. (2003). Análisis de la ejecución de lectura en una muestra de niños de primero a tercer grado de primaria: Un estudio comparativo.Tesis de Maestría, Facultad de Psicología, UNAM.

Flórez, R., Arias, N., \& Guzmán, R.J.(2006). El aprendizaje en la escuela: el lugar de la lectura y la escritura. Revista Educación y Educadores, 9, 118-133.

Greenwood, C. R., Dinwiddie, G., Terry, B., Wade, L., Stanley, S., Thibadeau, S., \& Delquadri, J. (1984). Teacher-versus-peer-mediated instruction: An ecobehavioral analysis of achievement outcomes. Journal of Applied Behavior Analysis, 17, 521-538.

Kohler, F. W., \& Greenwood, C. R. (1990). Effects of collateral peer supportative behaviors within the classwide peer tutoring program. Journal of Applied Behavior Analysis, 23, 307-322.

Leppänen, U., Niemi, P., Aunola, K., \& Nurmi, J. E. (2004). Development of reading skills among preschool and primary school pupils. Reading Research Quarterly,39 72-93.

López, A., \& Guevara, Y. (2008). Programa para prevención de problemas en la adquisición de la lectura y la escritura. Revista Mexicana de Análisis de la Conducta, 34, 57-78.

Macotela, S., Bermúdez, P., \& Castañeda, I. (1991). Inventario de Ejecución Académica: un Modelo Diagnóstico-prescriptivo para el Manejo de Problemas Asociados a la Lectura, la Escritura y las Matemáticas. México: Facultad de Psicología, U.N.A.M.

Mallette, B., Harper, G. F., Maheady, L., \& Dempsey, M. (1991). Retention of spelling words acquired using a peer-mediated instructional procedure. Education \& Training in Mental Retardation, 26, 156-164.

Mathes, P. G., Denton, C. A., Fletcher, J. M., Anthony, J. L., Francis, D. J., \& Schatschneider, C. (2005). The effects of theoretically different instruction and student characteristics on the skills of struggling readers. Reading Research Quarterly, 40, 148-182.

Pescador , J. A. (1983). Innovaciones para mejorar la calidad de la educación básica en México. Perfiles Educativos, 19, 28-42.

Sideridis, G. D, Utley, C., Greenwood, C. R., Delquadri, J., Dawson, H., Palmer, P., \& Reddy, S. (1997) Journal of Behavioral Education. 7, 435-462.

Vargas, J.D., \& Villares, L. (1999). Fundamentos y estrategias fundamentales de la guía tutela entre iguales en las primeras edades. Contextos Educativos, 2, 93106. 


\section{ANEXO 1}

\section{Ejemplo de listado de palabras usado por las maestras}

La siguiente lista formó parte de la evaluación durante la $3^{\mathrm{a}}$ semana de aplicación del CWPT. En esa semana las maestras trabajaron sobre el uso de la

"h", "r", "rr", "y", "Il".
1. hilo
11. sillón
2. llave
12. carretera
3. rayado
4. callejón
13. lluvia
14. corriente
5. zanahoria
15. rostizado
6. hormiga
16. carrocería
7. huarache
17. ferretería
8. cacahuate
18. amarillo
9. húmedo
19. zorrillo
10. hoja
20. sonrisa 
ANEXO 2

\section{Errores de regla}

Sustitución ortográfica (SO): Cambia letras por otras de sonido semejante, sin respetar reglas ortográficas. Ejemplo cuando escribe "b" por "v"; "g" por "j", etc.

Omisión ortográfica (OO): Omisión de la "h" en palabras como hoja, higo, etc. También incluye la omisión de la "u" en palabras como queso, juguete, etc.

Omisión de acentos (OA): El niño no coloca los acentos correspondientes.

Sustitución de mayúsculas por minúsculas y viceversa (SMm): El niño no respeta las reglas correspondientes al uso de mayúsculas en nombres propios, al inicio de un enunciado, o después de punto o punto y aparte. Asimismo, cuando el niño utiliza mayúsculas en palabras que no las requieren o en medio de la palabra, por ejemplo cuando escribe "gAto" en lugar de "gato".

\section{Errores específicos}

Adición (A): Agrega letras, sílabas o palabras. Por ejemplo escribe "higros" en lugar de "higos" o "Ella se come chocolate" en lugar de "Ella come chocolate".

Transposición (T): Traslada o cambia de lugar letras, sílabas o palabras. Por ejemplo, escribe "le" en vez de "el"; "ejonado" en vez de "enojado".

Omisión (O): Omite letras, sílabas o palabras. Por ejemplo escribe "kioco" en vez de " kiosco" o "El niño tomó" en lugar de "El niño lo tomó".

Sustitución (S): Cambia letras, sílabas o palabras, alterando el significado. Ejemplo escribe "pasos" en lugar de "pavos" o "Se te cayó" en lugar de "Se las entregó".

Inversión (I): Invierte la posición de las letras de igual orientación simétrica, escribiendo por ejemplo: "d. por "b"; "p" por "q"; "W" por "M". 